\title{
3D Reconstruction of the Human Jaw: A New Approach and Improvements
}

\author{
Moumen T. Ahmed, Ahmed H. Eid, and Aly A. Farag \\ Computer Vision and Image Processing Laboratory \\ University of Louisville, KY 40292 \\ moumen, ahmdhamd, farag@cvip.uofl.edu \\ http://www.cvip.uofl.edu
}

\begin{abstract}
This paper presents a new, practical approach for 3D reconstruction of the human jaw from a sequence of intra-oral images. This research has an immense value in various dental practices including implants, tooth alignment, and craniofacial surgery. Our approach is based on the recently-proposed space carving algorithm for shape recovery. This algorithm provides more flexibility to the reconstruction process and eliminates several constrains imposed by other traditional approaches such as stereo and shape from shading. Our experimental results have shown that the approach is able to reconstruct 3D models of the human jaw with sub-millimeter accuracy.
\end{abstract}

\section{Introduction}

Orthodontic treatment involves the application of force systems to teeth over time to correct malocclusion. In order to evaluate tooth movement progress, the orthodontist monitors this movement by means of visual inspection, intraoral measurements, fabrication of plastic models (casts), photographs and radiographs, a process which is both costly and time consuming. Obtaining a cast of the jaw is a complex operation for the orthodontist, an unpleasant experience for the patient and may not provide all the details of the jaw. Current technology in dental radiography can provide the orthodontist with $3 \mathrm{D}$ information of the jaw. While dental radiology is now widely accepted as a routine technique for dental examinations, the equipment is rather expensive and the resolution, being adequate for maxillofacial imaging, is still too low for 3D dental visualization. Furthermore, the dose required to enhance the resolution is unacceptably high. Some efforts have been devoted to computerized diagnosis in orthodontics, e.g., [27]. Usually, most of these 3D systems for dental applications found in the literature rely on obtaining an intermediate solid model of the jaw (cast or teeth imprints) and then capturing the $3 \mathrm{D}$ information from that model. User interaction is needed in such systems to determine the $3 \mathrm{D}$ coordinates of fiducial reference points on a dental cast. Other systems that can measure the 3D coordinates have been developed using either mechanical contact [8] or a traveling light principle 9 .

Our research lab has been involved for the last five years in a project, the jaw project, to develop a system for dentistry to go beyond traditional approaches 
in diagnosis, treatment planning, surgical simulation, and prosthetic replacements (1456]). Our ultimate goal is to transform a number of orthodontic protocols and maxillofacial practices from art into science. The jaw project builds a 3D model of the jaw, not from a cast, but from the actual human jaw. The system can obtain sequences of calibrated images of the upper/lower jaw using small intra-oral cameras, provide accurate 3D reconstruction from the acquired images and register robustly the $3 \mathrm{D}$ models built from multiple views. This research has an immense value in various dental practices including implants, tooth alignment, and craniofacial surgery. The research has also wide applications in teledentistry, dental education and training.

Our focus in this paper is on developing accurate, practical techniques to build 3D models of the human jaw. Several different approaches have been examined. The stereo approach has been by far the most widely used for shape recovery. However, one of the main problems of stereo is the correspondence problem. Due to the difficult nature of the correspondence problem, several constraints have been imposed on stereo. A large number of stereo techniques rely heavily on assumptions such as the existence of specific features in the images to produce satisfactory results. For example, featureless scenes are hard to reconstruct in stereo because corresponding points between the different images cannot be accurately found. Also, stereo is difficult to apply to images taken from arbitrary viewpoints. This is because corresponding image points become very hard to find if the images are taken from viewpoints far apart. As a consequence, stereo is inefficient approach for reconstruction of objects like human teeth, which, in addition to having many occlusion edges, have no specific geometric features. On the other hand, area-based stereo methods (also known as correlation-based methods) perform poorly due to the common homogeneous, textureless regions of the teeth. Figure 1 depicts a typical situation in which, feature-based stereo fails to deliver a sufficient number of correct matches (despite the use of a highly-robust method [10]). In addition, the constraints on the viewpoints make the stereo hard to apply for the jaw reconstruction since they are difficult to satisfy with the hand-held arm and CCD camera.

In our previous approach [15], we have successfully reconstructed the human jaw using the shape from shading (SFS) algorithm. However, since the SFS does not provide metric information, the reconstruction result from the SFS was fused with several range measurements to enhance its performance. These measurements help also in removing the ambiguity of the 3D visible surface discontinuities produced by the SFS. The depth measurements were obtained with the help of the digitizer arm and small laser projector mounted on the arm. The laser was employed to highlight the point in the captured image for which a depth measurement is acquired by the arm. The main drawback of this approach is its dependence on laser which, although being eye-safe, is unfavorable when human subjects are involved (actually it was a discouraging factor for some of our test patients). Moreover, the SFS algorithm would have problems with any tooth fillings due to the difference in albedo between tooth surfaces. 


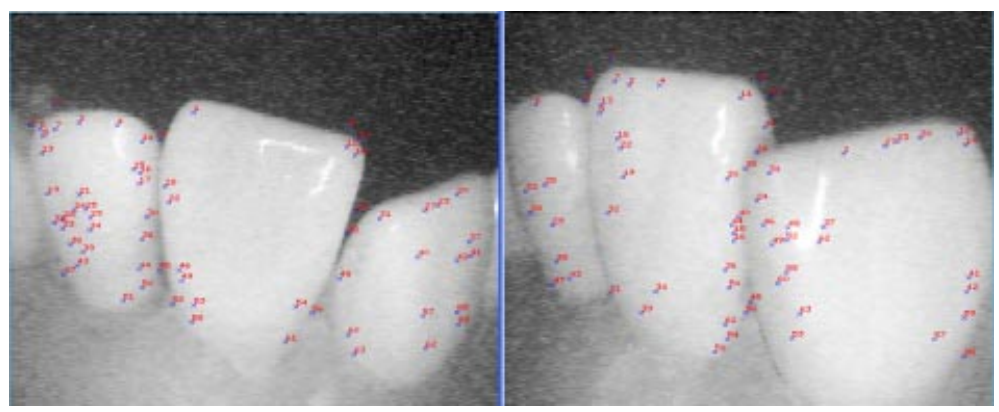

Fig. 1. Few correct and incorrect matches are obtained by a robust feature-based stereo [10] applied to a pair of jaw sequence: the labeled corresponding points, shown in crosses, were produced by Zhang's "image-matching" program after extraction of points of interest, doing correlation and rejection of some false matches using relaxation.

In this paper, we present an alternative $3 \mathrm{D}$ reconstruction method that does not require using the laser projector, any additional sensors or hardware. Yet this method recovers a complete, accurate 3D model of the jaw teeth from multiple views. Moreover, it alleviates the shortcomings of the stereo-based methods. On selecting this method, we depart from the traditional shape recovery approaches, e.g., stereo and SFS, which start off with images to find 3D surfaces, by employing a technique recently proposed by Kutulakos and Seitz called space carving [3]. Our choice for the space carving technique is guided by several concerns. Reconstruction using space carving does not rely on assumptions of the presence of specific image features, or on the positioning of the cameras, or the object geometry or texture. This technique allows us to reconstruct accurately 3D models that agree with the input images without the need to solve the difficult correspondence problem. In addition, since space carving is an "object-centered" reconstruction method, it becomes easy and straightforward to make use of any available a priori information on the shape of the object (which is true in our case), a key advantage of this method over the classical approaches. As such, this approach is more suitable and practical for our application.

This paper is organized as follows: Section II describes the overall system setup. Sections III presents our approach to tooth reconstruction using the space carving algorithm. Our experimental results are shown in Section IV followed by the concluding remarks in Section V.

\section{System Setup and Overview}

The experimental setup that we use consists of a MicroScribe $3 \mathrm{D}$ digitizer, an Ultra-Mirco CCD camera with a $5.5 \mathrm{~mm}$ lens, a regulated white light source, and an SGI Indigo2 machine that hosts the software required for data processing. For more details about the system hardware, please refer to [5], which uses the 
same setup except for the laser projector that we do not use in this paper on purpose.

The CCD camera is mounted on the stylus of the $3 \mathrm{D}$ digitizer and it has to be calibrated before its use. Because of the small focal lens of the camera, it suffers from some lens distortion (mainly radial distortion). The first coefficient of radial distortion is calibrated using a straight line-based technique $[12$ so that all acquired images can be undistorted before processing. After correcting for lens distortion, the camera can be safely modeled as an ideal pinhole camera, whose perspective projection matrix that encompasses the camera intrinsic and extrinsic parameters can be calibrated using a non-linear approach [11. If the camera is stationary, we do not have to re-calibrate again. Yet in the proposed system, the camera will be moving; This implies the recalculation of the perspective projection matrix. Being mounted on the digitizer arm, the camera location in the 3D space can be measured. Moreover, the arm provides the transformation that relates the new position and orientation of the camera to the world coordinate system. This transformation is used to update the camera extrinsic parameters and thus the camera perspective projection matrix. As such, the camera is maintained calibrated in all positions. In addition, the arm provides the position of the initial volume enclosing the tooth or any part of the jaw, which is to be carved by the space carving algorithm until the shape is reconstructed.

The five degrees of freedom provided by the arm enable the acquisition of a sequence of intra-oral images covering different parts of the jaw. Using the space carving algorithm, sets of voxels that represent the different parts of the jaw are computed. A fast registration technique [13] is employed to merge the resulting $3 \mathrm{D}$ models to obtain a complete $3 \mathrm{D}$ description of the jaw parts. The final stage transforms this model into patches of free form surfaces using a triangulation technique. This step enables the development of a 3D solid model for visualization. A cast can be fabricated from this model via rapid prototyping. Further processing that can be carried out on the digital model includes tooth separation, force analysis, implant planning, and surgical simulation.

\section{Space Carving}

In $3 \mathrm{D}$ object reconstruction, we attempt to achieve the reverse process of image formation by regenerating a $3 \mathrm{D}$ shape from various $2 \mathrm{D}$ projections. Space carving [3] attempts to produce the maximal 3D shape that is consistent with all the images. Space carving starts with an initial volume, $V$, that includes the object(s) to be reconstructed. This $3 \mathrm{D}$ space is then discretized into a finite set of voxels $v_{1}, v_{2}, \ldots, v_{n}$. The idea is to successively carve (remove) some voxels until the final 3D shape, $V^{*}$, agrees with all the input images. An outline of the algorithm is given below.

Space carving Algorithm:

Step 1: Initialize $V$ based on arm position and discretize it. 


\section{Step 2:}

- Determine the set of voxels $\operatorname{Vis}(V)$ on the surface of $V$.

- Project each voxel $v$ on $\operatorname{Vis}(V)$ to the different images where $v$ is visible.

- Determine the photo-consistency of each voxel $v$ on $\operatorname{Vis}(V)$.

Step 3: If no non-photoconsistent voxel is found, set $V^{*}=V$ and terminate. Otherwise, set $V=V$ - non-photoconsistent $v$ 's $\}$ and return to Step 2.

Each voxel on the surface of the volume, i.e., in $\operatorname{Vis}(V)$, is projected back to the different images using their respective projection matrices. To decide whether a voxel should be carved or not, the idea of color-consistency is used. The Lambertian model for the surface of the object is assumed. Under this model, light reflected from a single point on the surface of the object has the same intensity in all directions. Therefore, for a voxel to belong to the surface of the object, it must have the same color intensity, within some tolerance to allow for some light variations and some calibration inaccuracy, for all its projections to the different images provided. Voxels that are inconsistent with a single color, are viewed as free space in which different light rays intersect. By removing all color-inconsistent voxels, we are able to approximate a maximal photo-consistent shape that is defined by all the input images. The basic idea of space carving is illustrated in Figure 2. Three input images are used to generate the 3D model of the shape shown in the images. Voxels that project on the input images to pixels of similar color are kept and assigned that color. Voxels that project on the input images to pixels of different colors are removed.

Although the general idea in space carving is straightforward, modeling an algorithm to provide the desired results is not an easy task as the problem of occlusion must be treated. This is carefully taken care of in a multi-sweep fashion [3]. One important requirement by the algorithm is segmenting the objects from the background. This does not represent a problem with objects like teeth, since teeth are brighter than the interior of the mouth and have distinctive color from the gum. Therefore teeth can be easily segmented from the background in the image sequences.

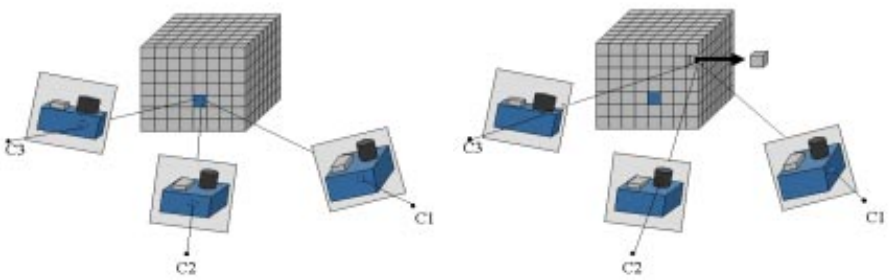

Fig. 2. Basic idea of space carving. Voxels are projected to the input images using their respective projection matrices. C1, C2 and C3 represent the optical centers of the three cameras. (a) Consistent voxels are assigned the color of their projections. (b) Inconsistent voxels are removed from the volume. 


\section{Experimental Results and Validation}

After calibrating the camera, sequences of images are captured for overlapping segments of the jaw. Each segment consists of about 3-5 images. The process of taking the images was relatively fast, taking less than a minute for each segment and a total of 10-12 minutes to cover the upper/lower jaw. The patient's jaw should not move during the acquisition time of each segment (less than a minute). However, movements between segments are permitted because the registration technique can align the individual segments. Figure 3 shows some images taken of a patient's jaw segment. We applied the space carving algorithm to the acquired images of each segment. The initial volume was selected as a cube and discretized into 70x70x70 voxels for a total of 343,000 voxels. Each segment is reconstructed after 4-5 passes of the space carving algorithm, which took about 16 seconds on an SGI Indigo2 machine. On average, the final volume contained 4,500 voxels. We used a $15 \%$ standard deviation threshold of the grayscale values to determine whether or not the voxels should be declared photo-inconsistent and consequently carved. This relatively high threshold was chosen in order to compensate for calibration errors, possible light changes from one image to another and any deviation from the Lamertian assumption (e.g., presence of specularities in some images). Figure 3 shows also the reconstructed result of one segment of the lower jaw from two different views. Once each segment of the jaw is reconstructed, they are registered [13] to compose the whole jaw, of which a part is shown in Figure 4 from two different views. The part shown in the figure took about 84 seconds of processing time for reconstruction and registration. Quantitative assessment and validation of the reconstruction is obtained by comparing some tooth measurements (e.g., height and width) from the reconstructed model to those of the real tooth as shown in Figure 5 The comparison showed accuracy within $0.47 \mathrm{~mm}$, which shows that the system can achieve sub-millimeter accuracy, similar to our previous approach but without the need for any range measurements.

\section{Conclusions and Future Extensions}

The $3 \mathrm{D}$ reconstruction of the human jaw has tremendous applications. To reconstruct the human jaw, we used the space carving algorithm which does not impose any constraints on the object geometry, the position of the camera, or the texture. This gives our approach a major, practical advantage over traditional shape recovery techniques such as SFS and stereo. Moreover, this algorithm successfully reconstructed 3D models of the human jaw with sub-millimeter accuracy, which is as accurate as our previous approach 5], but without using any range measurements or laser projectors. One key advantage of this new approach is the fact that it can easily exploit any available a prior information about the shape of the tooth. The algorithm can thus start with an initial volume whose shape is closer to the target object. This is expected to enhance and speed up the results of our approach. Our current research direction is directed to investigating this possibility. 


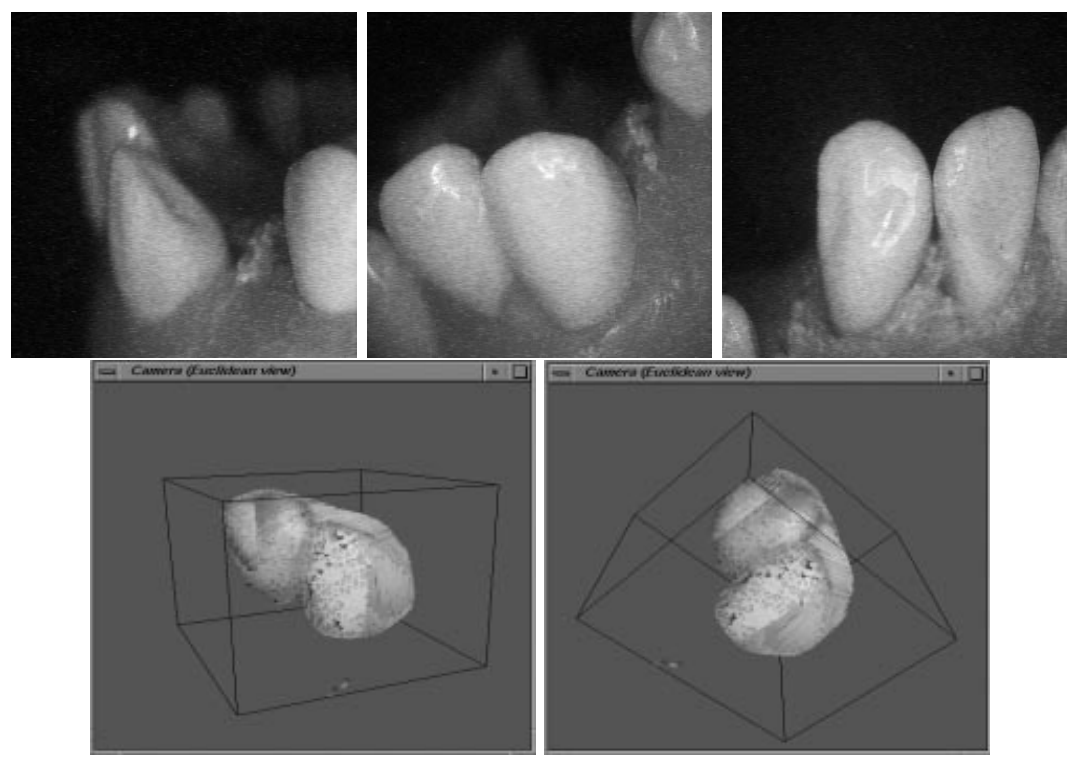

Fig. 3. Reconstruction results of a jaw segment consisting mainly of two teeth: (a)-(c) images of the two teeth acquired by the CCD camera. (d) and (e) The reconstructed teeth shown from two different views.

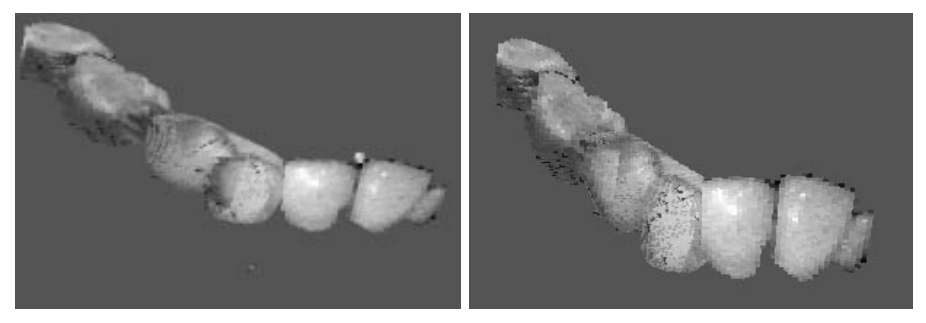

Fig. 4. Reconstructed part of the lower jaw shown from two views.
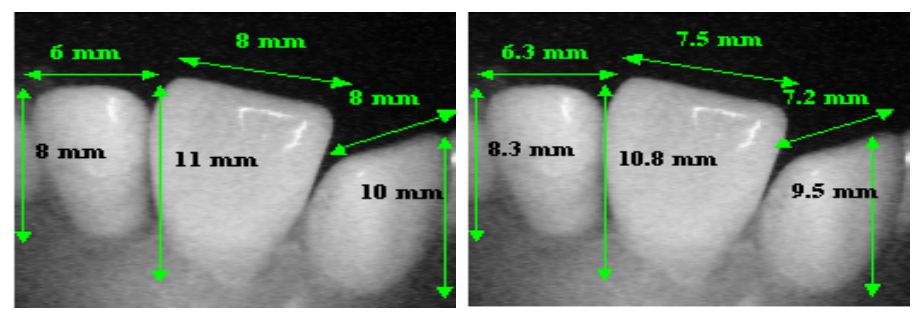

Fig. 5. Validation of the reconstructed model: (a) measurements from the real jaw, (b) measurements from the reconstructed model. 


\section{Acknowledgment}

This work was supported in part by grants from the Whitaker Foundation, and the NSF (ECS-9505674) institutions.

\section{References}

1. Sameh M. Yamany, Aly A. Farag, David Tasman and Allan G. Farman " A 3D Reconstruction System for Human Jaw Using a Sequence of Optical Images" IEEE Trans. on Medical Imaging,vol. 19(5), pp. 538-547, May 2000.

2. C. Bernard, A. Fournier, J. M. Brodeur, H. Naccache, and R. Guay, "Computerized diagnosis in orthodontics," Proc. 66th Gen. Session Int. Assoc. Dental Res. Montreal, 1988.

3. K. Kutulakos and S. Seitz. "Theory of Shape by Space Carving." Proceedings of IEEE International Conference On Computer Vision, Corfu, Greece, pp. 307-314, Sept. 1999.

4. M. Ahmed, S. M. Yamany, E. E. Hemayed, S. Roberts, S. Ahmed, and A. A. Farag, "3d reconstruction of the human jaw from a sequence of images," Proc. IEEE Computer Vision and Pattern Recognition Conf. (CVPR), Puerto Rico, pp. 646-653, June 1997.

5. S. M. Yamany, A. A. Farag, D. Tasman, and A. G. Farman, "A robust 3D reconstruction system for human jaw modeling," 2nd International Conference on Medical Image Computing and Computer-Assisted Intervention (MICCAI'99), Cambridge, England, pp. 778-787, Sept. 1999.

6. S. M. Yamany and A. A. Farag, "A system for human jaw modeling using intraoral images," Proc. IEEE Engineering in Medicine and Biology Society (EMBS) conference, Hong Kong 20, pp. 563-566, Oct. 1998.

7. D. Laurendeau and D. Possart, "A computer-vision technique for the acquisition and processing of 3D profiles of dental imprints: An application in orthodontics," IEEE Transactions on Medical Imaging 10, pp. 453-461, Sept 1991.

8. F. P. van der Linden, H. Boersma, T. Zelders, K. A. Peters, and J. H. Raben, "Three-dimensional analysis of dental casts by means of optocom," J. Dent. Res. 51(4), p. 1100, 1972.

9. A. A. Goshtasby, S. Nambala, W. G. deRijk, and S. D. Campbell, "A system for digital reconstruction of gypsum dental casts," IEEE Transactions on Medical Imaging 16, pp. 664-674, Oct. 1997.

10. Z. Zhang, R. Deriche, O. Faugeras, Q.-T. Luong, "A Robust Technique for Matching Two Uncalibrated Images Through the Recovery of the Unknown Epipolar Geometry," Artificial Intelligence Journal, 78, pages 87-119, Oct. 1995.

11. R. Klette, K. Schluns and A. Koschan, "Computer Vision: Three-Dimensional Data from Images," Springer, 1998.

12. B. Prescott and G. McLean, "Line-based correction of radial lens distortion," Graph. Models and Img. Process., 59(1), Jan. 1997.

13. S. M. Yamany and A. A. Farag, "Free-form surface registration using surface signatures," Proc. IEEE International Conference on Computer Vision (ICCV), Greece 2, pp. 1098-1104, Sept 1999. 